\title{
CENTRALIZERS OF ABELIAN SUBGROUPS IN LOCALLY FINITE SIMPLE GROUPS
}

\author{
by M. KUZUCUOĞLU
}

(Received 20th December 1994)

Dedicated to the memory of Brian Hartley

\begin{abstract}
It is shown that, if a non-linear locally finite simple group is a union of finite simple groups, then the centralizer of every element of odd order has a series of finite length with factors which are either locally solvable or non-abelian simple. Moreover, at least one of the factors is non-linear simple. This is also extended to abelian subgroup of odd orders.
\end{abstract}

1991 Mathematics subject classification: 20F50, $20 \mathrm{E} 32$.

\section{Introduction}

This article is concerned with the following two problems.

(1) Is it the case that in a non-linear locally finite simple group (LFS-group) the centralizer of every finite subgroup is infinite?

(2) Does the centralizer of every finite subgroup in a non-linear LFS-group involve an infinite non-linear simple group?

Theorem 1. Let $G$ be a non-linear LFS-group such that every finite set of elements of $G$ lies in a finite simple subgroup, and $x$ be an element of odd order $t$ in $G$. Then $C_{G}(x)$ has a series of finite length such that each factor is either locally solvable or non-abelian simple. Furthermore, at least one of the factors is a non-linear simple group.

As is discussed in more detail in [5], the centralizer of an element in a locally finite simple group is not necessarily of the above form because in Meierfrankenfeld groups of $V D A$ type the centralizer of every element is residually finite and involves an infinite simple group. If the centralizer of an element is residually finite and has the structure as in the above theorem, then it cannot involve an infinite simple group. See [11, Lemma 4.1].

Throughout this work, the only linear algebraic groups of classical type that we shall consider will be over fields of odd characteristic. We make this assumption because we use the results of $[12$, Chapter $I V$ on centralizers of unipotent elements in classical groups and these are valid only in good characteristic case. One of the major simplifications of this assumption arises because of the following theorem: 
If $G$ is a semisimple simply connected group with char $K$ good for $G$, then there is a bijective homeomorphism $f$ between the set $U$ of unipotent elements in $G$ and the set $V$ of the nilpotent elements of the Lie algebra of $G$. Moreover, $f$ commutes with the action of $G$ on $U$ and $V[13]$.

This theorem reduces the study of the centralizer of a unipotent element in $G$ to the study of the centralizer of a nilpotent element in the Lie algebra of $G$.

The case char $k=2$ might need a special treatment which we have not attempted.

We extend some of our results in [8] and [10] to more general groups. For this, we give some definitions. For the notation and definitions, see [8] and [10].

Definition 1. A finite abelian subgroup $F$ in a finite simple group $G$ of classical type or in an alternating group is called a nice group if whenever $G$ is of type $B_{\ell}$ or $D_{\ell}$, then $\mathrm{O}_{2}(F)$ is cyclic. If $G$ is an alternating group or of type $A_{\ell}$ or $C_{\ell}$, then every abelian subgroup is a nice group. In particular, every abelian group of odd order is a nice group.

Definition 2. A finite abelian subgroup $F$ contained in a finite simple group $G$ of classical type over a field of characteristic $p$ or in an alternating group is said to be of $\alpha-t y p e:$

(i) If $G$ is of classical type and $F$ is written as a direct product of a semisimple group $F_{s}$ and a unipotent group $F_{u}$, then $F_{s}$ is a nice abelian group in $G$ and the unipotent part $F_{u}$ is cyclic.

(ii) If $G$ is an alternating group, then every abelian subgroup is of $\alpha$-type.

In particular every abelian group of odd order in a classical group with cyclic unipotent part is an $\alpha$-type group.

Let $G$ be a countable simple LFS-group and $K$ be a Kegel sequence of $G$. A finite abelian group $F$ in $G$ is called a $K$-nice group if $F$ is a nice group in almost all Kegel components of the Kegel sequence $K$ of $G$.

Both questions in the introduction are answered affirmatively in [10] for the centralizer of a finite $K$-nice $K$ semisimple abelian subgroup in a non-linear LFSgroup. Here we extend the results of [10] to centralizers of $\alpha$-type subgroups.

Definition 3. A finite abelian group $F$ in a countably infinite LFS-group is said to be of $K-\alpha$-type if $F$ is an $\alpha$-type abelian subgroup in every Kegel component of a Kegel sequence $K=\left(G_{i}, M_{i}\right)$ of $G$. Moreover, for each $i,\left(\left|M_{i}\right|,|F|\right)=1$ and $M_{i}$ are solvable.

Theorem 2. If $F$ is a $K-\alpha$-type abelian subgroup of odd order in a non-linear countably infinite LFS-group $G$, then $C_{G}(F)$ has a series of finite length in which the factors are either non-abelian simple or locally solvable. Moreover, one of the factors is non-linear simple. 


\section{CENTRALIZERS OF ABELIAN SUBGROUPS IN FINITE SIMPLE GROUPS 219}

So we answer both of the questions affirmatively for $K-\alpha$-type abelian groups of odd order.

A reductive linear algebraic group $G$ is said to be of classical type if the simple components of the semisimple part of $G$ are all classical groups.

Theorem 3. Let $G$ be a connected reductive linear algebraic group of classical type over a field of characteristic $p \neq 2$. Let $\sigma$ be a Frobenius automorphism of $G$ and let $x$ be a unipotent element of order $p^{m}$ fixed by $\sigma$. Then $\left(C_{G}(x)\right)^{\sigma} \in T_{v p^{m}}$, where $v$ is the number of simple components of the semisimple part of $G$.

Recall that $[10,8], T_{n}$ consists of all locally finite groups having a series of finite length in which there are at most $n$ non-abelian simple factors and the rest are locally solvable.

Corollary 1. Let $X$ be a finite simple group of classical type over a field of characteristic $p \neq 2$ and $x$ be a unipotent element of order $p^{m}$ in $X$. Then $C_{X}(x)$ has a series in which there are at most $p^{m}$ non-abelian simple factors. Hence $C_{X}(x) \in T_{p^{m}}$. In particular the number of non-abelian simple factors in a series of $C_{X}(x)$ is bounded by the order of the unipotent element $x$.

Theorem 4. Let $X$ be a finite simple group of classical type over a field of characteristic $p \neq 2$ and $F$ be an abelian subgroup of $\alpha$-type of order $n p^{m}$ where $(n, p)=1$. Then $C_{X}(F) \in T_{p^{m} f(n)}$ where $f$ is a function from natural numbers to natural numbers independent of $X$.

\section{Proof of the results}

Lemma 1 [8]. (i) The classes $T_{n}$ are closed under taking normal subgroups and quotients.

(ii) If $M \triangleleft G, M \in T_{m}$ and $G / M \in T_{n}$, then $G \in T_{m+n}$.

Lemma 2. Let $G$ be a linear algebraic group and $x$ be an element of $G$ fixed by the Frobenius automorphism $\sigma$ of $G$ and assume that $G / G^{0}$ is solvable. Then $\left(C_{G}(x)\right)^{\sigma} \in T_{n}$, iff $\left(C_{G^{0}}(x)\right)^{\sigma} \in T_{n}$.

Proof. For $\left(C_{G^{0}}(x)\right)^{\sigma}=\left(C_{G}(x)\right)^{\sigma} \cap G^{0}$, and so $\left(C_{G}(x)\right)^{\sigma} /\left(C_{G^{0}}(x)\right)^{\sigma}$ is isomorphic to a subgroup of the solvable group $G / G^{0}$. Hence Lemma 1 gives the result.

Lemma 3. Let $G$ be a linear algebraic group and $\sigma$ be a Frobenius automorphism of $G$, and $N$ be a $\sigma$-invariant locally finite subgroup of $G$ contained in the centre. Let $x$ be any $\sigma$-invariant element of $G$. Then

$$
\left(C_{G}(x)\right)^{\sigma} \in T_{n} \quad \text { iff } \quad\left(C_{G / N}(x)\right)^{\sigma} \in T_{n}
$$


i.e. the number of non-abelian simple factors in a series of $\left(C_{G}(x)\right)^{\sigma}$ and $\left(C_{G / N}(x)\right)^{\sigma}$ do not change.

Proof. Let

$$
\left(C_{G / N}(x)\right)^{\sigma}=C / N=\left\{g N \in G / N \mid g^{-1} g^{\sigma} \in N \quad \text { and } \quad[g, x] \in N\right\} .
$$

By using the fact that $N$ is in the centre, it is easy to see that $\left(C_{G}(x)\right)^{\sigma} \triangleleft C$. Now we claim that $C /\left(C_{G}(x)\right)^{\sigma}$ is abelian. Indeed for any $h, g \in C$

$$
g^{-1} g^{\sigma}=z, \quad h^{-1} h^{\sigma}=w, \quad[g, x]=u, \quad[h, x]=v
$$

where $z, w, u, v \in N$. Hence

$$
[g, h]^{\sigma}=\left[g^{\sigma}, h^{\sigma}\right]=\left[g g^{-1} g^{\sigma}, h h^{-1} h^{\sigma}\right]=[g z, h w]=[g, h] .
$$

Now using Witt's identity and the fact that $N \leq Z(G)$ we get $[[g, h], x]=e$.

This implies, by Lemma 1 , that the number of non-abelian simple factors in a series of $C$ and in a series of $C_{G}(x)^{\sigma}$ are equal as $C / N \in T_{n}$ iff $C \in T_{n}$.

Proof of Theorem 3. It is well known that $G=Z G^{\prime}$ where $G^{\prime}$ is the commutator subgroup of $G$ and $Z$ is a central torus. $G^{\prime}$ is a connected semisimple linear algebraic group and $Z$ is a $p^{\prime}$-group. Hence the unipotent element $x \in G$ is contained in the semisimple part $G^{\prime}$. Then we get $C_{G}(x)=Z C_{G^{\prime}}(x)$. The group $Z$ is a connected $\sigma$-invariant central subgroup of $G$ and $C_{G}(x)$ is a $\sigma$-invariant subgroup of $G^{\prime}$. Then $\left(C_{G}(x)\right)^{\sigma}=\left(Z C_{G}(x)\right)^{\sigma}$.

$\left(C_{G^{\prime}}(x)\right)^{\sigma}=\left(C_{G}(x)\right)^{\sigma} \cap G^{\prime}$. Then $\left(C_{G}(x)\right)^{\sigma} /\left(C_{G^{\prime}}(x)\right)^{\sigma}$ is isomorphic to a subgroup of the abelian group $G / G^{\prime}$. Since we are interested in the number of non-abelian simple factors of the series of $\left(C_{G}(x)\right)^{\sigma}$, by Lemma 1 it is enough to find the number of nonabelian simple factors of $\left(C_{G^{\prime}}(x)\right)^{\sigma}$.

The group $G^{\prime}$ is a connected semisimple linear algebraic group. Hence $G^{\prime}$ can be written as a central product of say $v$ simple linear algebraic groups. By assumption all simple components of $G^{\prime}$ are of classical type. Let $G^{\prime}=G_{1} \ldots G_{v}$

$$
\left(G^{\prime}\right)^{\sigma}=\left(G_{1} G_{2} \ldots G_{v}\right)^{\sigma}
$$

where each $G_{i}$ is a simple linear algebraic group of classical type. Let $Z=Z\left(G^{\prime}\right)=$ $Z\left(G_{1}\right) \ldots Z\left(G_{v}\right)$. Then

$$
G^{\prime} / Z=\bar{G}=\bar{G}_{1} \ldots \bar{G}_{v} \text { and } \bar{G}_{i}=G_{i} / Z_{i}
$$

By the Krull Schmidt theorem $\sigma\left(G_{i} Z\right)=G_{j} Z$, then by taking the derived group we see that

$$
\sigma\left(G_{i}\right)=\sigma\left(\left(G_{i} Z\right)^{\prime}\right)=\left(G_{j} Z\right)^{\prime}=G_{j}
$$


Therefore $\sigma$ permutes the $G_{i}$ 's. Let $O_{i}, i=1,2, \ldots, r$ be the orbits of $\sigma$ on $\left\{G_{1}, \ldots, G_{v}\right\}$ and let $K_{i}=\Pi_{D \in O_{i}} D$. Hence $G^{\prime}$ is the central product of $K_{1} K_{2} \ldots K_{r}$. Let $\bar{K}$ be any one of the orbits of $\sigma$ on $\bar{G}$; say for simplicity the one containing $\bar{G}_{1}$

$$
\bar{K}=\bar{G}_{1} \sigma\left(\bar{G}_{1}\right) \ldots \sigma^{t(1)-1}\left(\bar{G}_{1}\right)
$$

and $\sigma^{(1)}\left(\bar{G}_{1}\right)=\bar{G}_{1}$. Then $\bar{K}$ is the direct product of the group $\sigma^{i}\left(\bar{G}_{1}\right)$ and

$$
\bar{K}^{\sigma}=\left\{c_{0} \sigma\left(c_{1}\right) \ldots \sigma^{t(1)-1}\left(c_{t(1)-1}\right) \mid \sigma\left(c_{0} \sigma\left(c_{1}\right) \ldots \sigma^{t(1)-1}\left(c_{t(1)-1}\right)\right)=c_{0} \sigma\left(c_{1}\right) \ldots \sigma^{t(1)-1}\left(c_{t(1)-1}\right)\right\},
$$

where $c_{i} \in \bar{G}_{1}$. This implies that $c_{i}=c_{0}$ for all $i=1, \ldots, t(1)-1$. Hence

$$
\bar{K}^{\sigma}=\left\{c \sigma(c) \ldots \sigma^{t(1)-1}(c) \mid c \in \bar{G}_{1}, \quad \sigma^{t(1)} c=c\right\} \cong C_{\bar{G}_{1}}\left(\sigma^{t(1)}\right)
$$

Therefore

$$
\left(G^{\prime} / Z\right)^{\sigma} \cong C_{\bar{G}_{1}}\left(\sigma^{t(1)}\right) \times \cdots \times C_{\bar{G}_{i}}\left(\sigma^{t(i)}\right) \text { where } i \leq v
$$

The unipotent element $x$ of order $p^{m}$ with which we have started off determines the element

$$
x Z \in\left(G^{\prime} / Z\right)^{\sigma} \cong C_{\bar{G}_{1}}\left(\sigma^{t(1)}\right) \times \cdots \times C_{\bar{G}_{i}}\left(\sigma^{t(i)}\right)
$$

Let $\left(x_{1} Z_{1}, x_{2} Z_{2}, \ldots, x_{i} Z_{i}\right)$ be the unipotent element under the above isomorphism such that $\left(x_{j} Z_{j}\right)^{\sigma^{(t)}}=x_{j} Z_{j}$. Hence

$$
C_{\left(G^{\prime} / Z^{\sigma}\right.}(x) \cong C_{\left(\bar{G}_{1}\right) \sigma^{\alpha(1)}}\left(x_{1}\right) \times \cdots \times C_{\left(\bar{G}_{i}\right) \sigma^{\sigma(i)}}\left(x_{i}\right)
$$

observe that $i \leq v$. Now by Lemma 3, if we find $C_{(G / Z)^{\circ}}(x) \in T_{p^{m}}$, then we say $\left(C_{G}(x)\right)^{\sigma} \in T_{p^{m}}$. Since the number of factors is $\leq v$ we find in each factor and multiply it with $i \leq v$. Since $\sigma^{r(i)}$ is also a Frobenius automorphism replace $\sigma^{t(i)}$ with $\sigma$. Then the question reduces to:

Let $G$ be a classical type simple group over a field of characteristic $p \neq 2$ and $x$ be a unipotent element of order $p^{m}$ in $G$. Then $C_{\sigma^{\sigma}}(x)=\left(C_{G}(x)\right)^{\sigma} \in T_{p^{m}}$.

So we answer this question for each classical type. If we show $\left(C_{G L_{n}}(x)\right)^{\sigma} \in T_{p^{m}}$, then by similar arguments as before $\left(C_{S L_{n}}(x)\right)^{\sigma} \in T_{p^{m}}$. Now by Lemma 3 we have

$$
\left(C_{\left(S L_{n} / Z\right)}(x)\right)^{\sigma}=C_{\left(S L_{n} / Z\right)^{a}}(x) \in T_{p^{m}}
$$

and we are done.

So we show that $\left(C_{G L_{n}}(x)\right)^{\sigma} \in T_{p^{m}}$.

Let $G=G L_{n}$. By $[12,1.7$ and 1.8] we get

$$
\left(C_{G L_{n}}(x)\right)^{\sigma}=\left(R \prod_{i=1}^{p^{m}} G L_{r_{i}}\right)^{\sigma}=(R C)^{\sigma}, \quad C \cong \prod G L_{r_{i}},
$$


and $C$ is a reductive group and the product is a semidirect product. $R$ and $C$ are defined over the field $k$ hence they are $\sigma$ invariant. If $x y \in(R C)^{\sigma}, x \in R, y \in C$, then $(x y)^{\sigma}=x^{\sigma} y^{\sigma}=x y$ which implies that $x^{-1} x^{\sigma}=y\left(y^{-1}\right)^{\sigma} \in R \cap C=\{e\}$ as both $R$ and $C$ are invariant $x^{\sigma}=x, y^{\sigma}=y$. So $(R C)^{\sigma}=R^{\sigma} C^{\sigma}$ since $R$ is the unipotent radical it is enough to find the number of non-abelian simple factors of $C^{\sigma}$. But

$$
\begin{aligned}
C^{\sigma} & =\left(G L_{r_{1}} \times G L_{r_{2}} \times \ldots G L_{r_{i}}\right)^{\sigma} \\
& =\left(G L_{r_{1}}\right)^{\sigma^{\sigma(1)}} \times \ldots \times\left(G L_{r_{j}}\right)^{\sigma^{\sigma(1)}} \in T_{p^{m}}
\end{aligned}
$$

since by $[12,1.7,1.8]$, the number of $r_{i}$ 's is less than or equal to $p^{m}$. Observe that the only ones $r_{i} \geq 2$ might give a non-abelian simple factor. Hence we are done for this case.

Now let $G$ be an orthogonal, unitary or a symplectic group. Then by [12, Theorem 2.23, page 260] $C_{G}(x)=R C$ where $R$ is the unipotent radical of $C_{G}(x)$ and $C$ is a connected reductive linear algebraic group and the product is the semidirect product of $C$ and $R$. Then by [12, Theorem 2.25] $C(k)$ is isomorphic to

(i) $\prod_{i=1}^{p^{m}} U\left(h_{i}, k\right)$ in the unitary case

(ii) $\prod_{i=1, i \text { even }}^{p^{m}} S p_{r_{i}}(k) \times \prod_{i=1, i \text { odd }}^{p^{m}} O\left(h_{i}, k\right)$ in the orthogonal case

(iii) $\prod_{i=1}^{p^{m}} S p_{r_{i}}(k) \times \prod_{i=1}^{p^{m}} O\left(h_{i}, k\right)$ in the symplectic case.

Now as in the case of $G L_{n}$ we have $\left(C_{G}(x)\right)^{\sigma}=(R C)^{\sigma}=R^{\sigma} C^{\sigma}$ and it is enough to find the number of non-abelian composition factors of a series of $(C)^{\sigma}$. Since the number of direct factors is less than or equal to $p^{m}$ and each direct factor gives at most one non-abelian simple factor to $(C)^{\sigma}$ (in case $r_{i} \geq 2$ ) we get $C^{\sigma} \in T_{p^{m}}$. Observe that the simple factors might be the twisted types of the given groups.

Proof of Theorem 4. Let $F=F_{s} F_{u}$ where $F_{s}$ be the semisimple subgroup of order $n$ and $F_{u}$ be the cyclic unipotent of order $p^{m}$. Every finite simple group $X$ of Lie type can be obtained as $X=O^{p^{\prime}}\left(G^{\sigma}\right)$ where $G$ is a simple linear algebraic group of adjoint type over an algebraically closed field of characteristic $p$, and $\sigma$ is a Frobenius automorphism of $G$. Then $F$ can be considered as a subgroup of $G$. Since $F$ is an $\alpha$-type abelian group by [12, Theorem 5.8(c)] there exists a $\sigma$ invariant maximal torus of $G$ containing $F_{s}=\left\langle a_{1}, \ldots, a_{r}\right\rangle$. Then by [12, Theorem 4.1$]$.

$$
C_{G}\left(F_{s}\right)=<T, X_{\beta}, n_{w} \mid \beta\left(a_{i}\right)=1, \beta \in \Phi, a_{i}^{w}=a_{i}, \quad i=1,2, \ldots r>
$$

and

$$
C_{G}\left(F_{s}\right)^{0}=<T, X_{\beta}, \mid \beta\left(a_{i}\right)=1, \beta \in \Phi, \quad i=1,2, \ldots r>
$$


$C_{G}\left(F_{s}\right)^{0}$ is a connected reductive group and it is of classical type. (One can extract this from [8, Construction 4.1].) Since for any semisimple element $a_{i} \in F$ by [12, Corollary 4.4] $C_{G}\left(a_{i}\right) / C_{G}\left(a_{i}\right)^{0}$ is abelian we get that $C_{G}\left(F_{s}\right) / C_{G}\left(F_{s}\right)^{0}$ is abelian.

We need to find $C_{G}(F)^{\sigma}$. But $C_{G}(F)^{\sigma}=\left(C_{G}\left(F_{s}\right) \cap C_{G}\left(F_{u}\right)\right)^{\sigma}=\left(C_{C_{G}\left(F_{s}\right)}(x)\right)^{\sigma}$ where $F_{u}=\langle x\rangle$. In order to find the number of non-abelian simple factors in a series of $\left(C_{C_{G}\left(F_{5}\right)}(x)\right)^{\sigma}$, by Lemma 2 it is enough to find the number of non-abelian simple factors in a series of $\left(C_{C_{G}\left(F_{5}\right)^{0}}(x)\right)^{\sigma}$. Since $C_{G}\left(F_{s}\right)^{0}$ is a connected reductive linear algebraic group and $x$ is a unipotent element of $C_{G}\left(F_{s}\right)^{0}$ we have by Theorem $3\left(C_{\left.C_{G\left(F_{s}\right)^{0}}(x)\right)^{0} \in T_{p^{m} f(n)}}\right.$ where $f$ is a function from natural numbers to natural numbers independent of the group $X$ (see [10, Theorem 3]). One can see that the number of simple components of the semisimple part of $C_{G}\left(F_{s}\right)^{0}$ is $f(n)$ from [10, Theorem 3]. Hence

$$
\left(C_{G}(F)\right)^{\sigma}=\left(C_{C_{G}\left(F_{5}\right)}(x)\right)^{\sigma} \in T_{p^{m}(n)} .
$$

Now

$$
C_{X}(F)=C_{o^{\prime}\left(G^{o}\right)}(F)=C_{G^{o}}(F) \cap O^{p^{\prime}}\left(G^{\sigma}\right) .
$$

Since $O^{p^{\prime}}\left(G^{\sigma}\right)$ is normal in $G^{\sigma}$, we have $C_{o^{\prime}\left(G^{o}\right)}(x) \triangleleft C_{G^{o}}(x)$. Hence by Lemma 1 $C_{o^{\prime}\left(G^{\circ}\right)}(F) \in T_{p^{m} /(n)}$.

Remark. Let $G$ be a simple linear algebraic group of classical type over a field of odd characteristic $p$ and $F$ be an abelian subgroup of $G$ with cyclic unipotent part $F_{u}$ and semisimple part $F_{s}$. Let $F_{u}=\langle x\rangle$ and $\left|F_{u}\right|=p^{m}$. Assume that $G$ has rank $l$. Let $r$ be a given integer. To see that when $l$ is sufficiently large $\left(C_{G}(F)\right)^{\sigma}$ involves simple classical groups of rank greater than or equal to $r$, we need to spell out some of the facts from [12, Chapter $I V]$. In fact by [10, Theorem 3] if $|F|=n p^{m}$ where $\left|F_{s}\right|=n$ $(n, p)=1$, then we have $C_{G}\left(F_{s}\right) \in T_{f(n)}$. So it is enough to say that at least one of the numbers $r_{i}$ in the Theorem 3 is greater than or equal to $r$. The numbers $r_{i}$ 's arise in the following way. (See [12, Chapter IV].)

For simplicity we describe it when $G=G L$. Let $V$ be a vector space over a field $k$ of dimension $n$ and $x$ be a nilpotent linear transformation on $V$ such that $x^{d}=0$. Let $A(X)$ be the $k$-algebra of linear transformations of $V$ generated by $x$. Then $A(X)=k[T] / f k[T]$ where $f$ is the minimal polynomial of $x . V$ considered as an $A(X)$ module is denoted by $V(X)$ and by [12] $V(X) \cong \oplus_{i=1}^{s} M\left(d_{i}\right)$ where $M\left(d_{i}\right)$ is the $A$-module $x^{d-d_{i}} A$. By [12, Chapter $\left.I V\right]$ there exists $e_{i}(1 \leq i \leq s)$ in $V$ and integers $d_{i}$ such that $x^{d_{i}} e_{i}=0$ and that $x^{j} e_{i}\left(0 \leq j<d_{i}, 1 \leq i \leq s\right)$ is a $k$ basis of $V$. In Corollary $1.8 r_{j}$ is the number of $d_{i}$ equal to $j$. There is a $k$ isomorphism $\phi: C \rightarrow \prod G L_{r_{i}}$. So in case $G=G L$ if $l>r\left(p^{m}(f(n))\right)$ where $f$ is as in [10, Theorem 3] we have at least one of the $r_{i} \geq r$.

For the other classical groups one can extract from 2.19 and 2.25 that if $l>r\left(3 p^{m} f(n)\right)$, then $\left(C_{G}(F)\right)^{\sigma}$ involves a classical simple group of rank greater than or equal to $r$. 
Proof of Theorem 1. Let $\pi$ be the set of prime divisors of $t$. By using the classification of the finite simple groups we may assume that every finite subgroup of $G$ is contained either in an alternating group or in one of the families $A, B, \ldots,{ }^{2} G_{2}$. Since the number of families is finite one can show that there exists a fixed family such that every finite subset of $G$ is contained in a simple group of fixed type.

The alternating case, for an arbitrary element is done in [8, Lemma 2.5]. If every finite subgroup is contained in a linear group with bounded rank parameter, then by [7], [2] and [15] the group should be linear. But we assumed that $G$ is non-linear, hence every finite subgroup of $G$ should be contained in a classical group of fixed type with unbounded rank parameter.

If there exists a prime $p$ such that every finite subset of $G$ is contained in a finite classical group of fixed type over a field of characteristic $p$, then we have either $p \nmid t$ or $p \mid t$.

If $p \nmid t$, then $x$ becomes a semisimple element in every group containing $x$. Thus as in [8, Theorem B] every finite set of elements of $C_{G}(x)$ lies in a subgroup of the form $C_{H}(x)$ where $H$ is a finite simple group of fixed classical type and $x$ is a semisimple element of $H$. Hence by [8, Lemma 2.3] we are done.

If $p \mid t$, then by Theorem 4 we have $C_{G}(x)$ is locally $T_{p^{m} f(n)}$ where $t=p^{m} n,(n, p)=1$. Again by [8, Lemma 2.3] we are done. Note that as $x$ is an element of odd order we do not need the centralizers of 2 elements in classical groups over fields of characteristic 2 .

If no such prime $p$ exists, then for each prime $q$ in $\pi$ there is a finite subset of $G$ that is not contained in the above type group over a field of characteristic $q$ and hence there is a finite subset $F$ and $G$ containing $x$ such that $x$ is a semisimple element in every group containing $F$. Hence every finite set of elements of $C_{G}(x)$ lies in a subgroup of the form $C_{H}(x)$ where $H$ is as in the above form and $x$ is a semisimple element in $H$. Again by [8, Lemma 2.3] we are done.

Proof of Theorem 2. Let $F$ be a $K-\alpha$-type abelian subgroup of odd order and $K=\left(G_{i}, M_{i}\right)$ be the given Kegel sequence of $G$. If necessary by passing to a subsequence we may assume that $G_{i} / M_{i}$ are all alternating or all belong to a fixed type classical family. If they are all alternating, then by [10, Theorem 1] we are done. Assume that $G_{i} / M_{i}$ all belong to a fixed classical family over a field of characteristic $p$. Then by Theorem 4 we have $C_{G_{i} / M_{i}}(F) \in T_{p^{m} f(n)}$. Since by assumption $\left(\left|M_{i}\right|,|F|\right)=1$ we have $C_{G_{i} / M_{i}}(F)=C_{G_{i}}(F) M_{i} / M_{i}$. Since $M_{i}$ are all solvable and $C_{G_{i}}(F) M_{i} / M_{i} \cong C_{G_{i}}(F) /\left(C_{G_{i}}(F) \cap M_{i}\right)$ we get $C_{G_{i}}(F) \in T_{p^{m} /(n)}$ for all $i$. Now by [10, Lemma 3(ii)] we get $C_{G}(F) \in T_{p^{m} f(n)}$.

By the remark after the proof of Theorem $4 C_{G_{i}}(F)$ involves alternating groups of unbounded orders and $C_{G}(F)$ has a series of finite length in which each factor is either non-abelian simple or locally solvable, one of the factors of the series of $C_{G}(F)$ must be non-linear.

Acknowledgement. I would like to thank Prof. Brian Hartley for many fruitful conversations and for reading a previous version of this work. I also would like to 
thank the Scientific and Technical Research Council of Turkey and the Royal Society of the United Kingdom for support.

\title{
REFERENCES
}

1. V. V. BeLYAEV, Locally finite Chevalley groups, Studies in Group Theory (Acad. of Sciences of the U.S.S.R. Urals Scientific Centre, 1984).

2. A. V. Borovik, Embeddings of finite Chevalley groups and periodic linear groups, Sibirsky, Mat. Zh. 24 (1983), 26-35.

3. R. W. CARTER, Simple groups of Lie Type (John Wiley London, 1972).

4. R. W. CARTER, Finite groups of Lie Type (John Wiley London, 1985).

5. B. Hartley, On some simple locally finite groups constructed by Meierfrankenfeld, $J$. London Math. Soc. (2) 52 (1995), 345-355.

6. B. HaRtLeY, Centralizing properties in simple locally finite groups and large finite classical groups, J. Austral. Math. Soc. Ser. A 49 (1990), 502-513.

7. B. Hartley and G. Shute, Monomorphisms and direct limits of finite groups of Lie type, Quart. J. Math. Oxford(2) 35 (1984), 49-71.

8. B. HaRtley and M. KuzucuoĞLu, Centralizers of elements in locally finite simple groups, Proc. London Math. Soc. (3) 62 (1991), 301-324.

9. O. H. Kegel and B. Wehrfritz, Locally Finite Groups (North Holland, Amsterdam, 1973).

10. M. KuzucuoăLu, Centralizers of semisimple subgroups in locally finite simple groups, Rend. Sem. Mat. Univ. Padova 92 (1994), 79-90.

11. M. KuzucuoĞLu, Barely Transitive Permutation Groups, Arch. Math. 55 (1990), $521-532$.

12. T. A. SPR INGer and R. Steingerg, Conjugacy Classes, in Seminar on Algebraic Groups and Related Finite Groups (Lecture Notes in Math. Vol. 131, Springer Verlag, Berlin Heidelberg New York, 1970).

13. T. A. SPRInger, Proceedings Symposia Pure Mathematics Vol. 29 (Providence R.I. American Mathematical Society, 1975), 373-391.

14. R. Steinberg, Endomorphism of Algebraic Groups (Mem. Amer. Math. Soc. No. 80, American Math. Soc. Providence, R.I., 1968).

15. S. Thomas, The classification of the simple periodic linear groups, Arch. Math. 41 (1983), 103-116.

\author{
Department of Mathematics \\ Middle East Technical University \\ 06531, AnKara, Turkey \\ E-mail address: matmah@rorqual.cc.metu.edu.tr
}

